\title{
Metabolic and Haematological Profile of Infants Born to Gestational and Pregestational Diabetic Mothers
}

\author{
SHEIKH FARJANA SONIA ${ }^{1}$, MD. FAZLUL HAQUE², RUMA PARVIN ${ }^{3}$, AZMERI SULTANA $^{3}$, SHARMIN \\ AFROZE ${ }^{4}$, MOHAMMAD SAYEED HASSAN 5
}

\begin{abstract}
Background: Diabetes mellitus is the most common metabolic disorder complicating pregnancy. Infants of diabetic mothers (IDMs) are prone to develop complications during early neonatal period. The study was undertaken to describe and compare the metabolic and haematological parameter of infants born to gestational (GDM) and pregestational (PGDM) diabetic mothers.
\end{abstract}

Meterials and Methods: The hospital based prospective study was conducted at special care neonatology unit of Dr. M. R. Khan Shishu Hospital \& ICH from January 2018 to December 2018. Blood samples were collected to perform relevant biochemical and haematological tests.

Results: Of the 68 neonates, $61.8 \%$ were born to mothers with GDM and $38.2 \%$ were born to mothers with PGDM. In case of GDM, $4.8 \%$ were Small for Gestational Age (SGA), 61.9\% were Appropriate for Gestational Age (AGA) and 33.3\% were Large for Gestational Age (LGA). Hypoglycemia occurred in $71.4 \%$ of neonates with GDM and $53.8 \%$ of neonates with PGDM. Hypocalcemia was observed in $47.6 \%$ of neonates with GDM and $69.2 \%$ of neonates with PGDM. Frequency of hypoglycemia, polycythemia and hyperbilirubinemia were significantly higher in neonates born to mothers with GDM ( $p$ value 0.01, 0.02 and $<0.001$ respectively) and frequency of hypocalcemia was significantly higher in neonates born to mothers with PGDM ( $p$ value 0.02).

Conclusions: The frequency of hypoglycaemia, polycythemia and hyperbilirubinemia were significantly more in newborns of GDM compared to infants of PGDM but the frequency of hypocalcemia was significantly more in newborns of PGDM.

Keywords: Gestational diabetic mother (GDM), Hypoglycemia, Hypocalcemia, Hyperbilirubinemia, Polycythemia, Pregestational diabetic mother (PGDM)

\section{Introduction:}

Diabetes mellitus (DM) is the most common medical complication in pregnancy. It is a chronic metabolic disorder due to either insulin deficiency (relative or absolute) or due to peripheral tissue resistance to the action of insulin. ${ }^{1}$ Pregnant women are divided

1. Assistant Professor, Dept. of Paediatrics, Dr. M. R. Khan Shishu Hospital \& ICH

2. Professor of Paediatrics \& Director (Academic), Dr. M. R. Khan Shishu Hospital \& ICH

3. Associate Professor, Dept. of Paediatrics, Dr. M. R. Khan Shishu Hospital \& ICH

4. Assistant Professor, Dept. of Neonatotology, Dr. M. R. Khan Shishu Hospital \& ICH

5. Assistant Professor, Dept. of Neuromedicine, National Institute of Neuroscience

Correspondence: Dr. Sheikh Farjana Sonia, Assistant Professor, Dept. of Paediatrics, Dr. M. R. Khan Shishu Hospital \& ICH. Cell: +8801752038848, E-mail: soniafarzana7@gmail.com

Received: 19/06/2019 into those who were known to have diabetes before pregnancy as pregestational diabetic mother (PGDM) , and those diagnosed during pregnancy as gestational diabetic mother (GDM). ${ }^{2}$ About $1-14 \%$ of all pregnancies are complicated by diabetes mellitus, $90 \%$ of them are GDM. ${ }^{1}$ GDM normally occurs during $2^{\text {nd }}$ trimester of pregnancy (24-28 weeks), mother at higher risk may affects earlier.

World Health Organization (WHO) has predicted that between 1995 and 2025, there will be a 35\% increase in the world wide prevalence of diabetes. ${ }^{3}$ Moreover, women born in Asian countries display the highest prevalence of GDM, with upto $17 \%$ of women likely to develop GDM in comparison to $4 \%$ of European and white American women. ${ }^{4,5}$ According to the $7^{\text {th }}$ International Diabetes Federation Diabetes Atlas in 2015, 20.9 million (16.2\%) live births were affected 
with hyperglycemia in pregnancy \& estimated $85.1 \%$ of those cases were because of GDM. ${ }^{6}$ Local studies show that prevalence rates of GDM were between $6 \%$ and $14 \%$ in Bangladesh. 7,8 Maternal and fetal perinatal mortality was also high among GDM mothers in Bangladesh. ${ }^{9}$

IDMs are at risk of several metabolic complications like hypoglycemia, hypocalcemia and hematological complications like polycythemia, hyoerbilirubinemia etc. With the advent of insulin and oral hypoglycemic agents, outcome of pregnant women with diabetes has improved but abnormal fetal metabolism during pregnancy complicated by maternal diabetes mellitus results in multiple neonatal sequelae, including abnormalities of growth, cardiorespiratory function, bilirubin metabolism and congenital anomalies. ${ }^{10}$

During pregnancy, elevated maternal serum glucose results in elevated fetal serum glucose. Because insulin does not cross the placenta, fetal hyperglycemia results in pancreatic stimulation \& subsequent hyperplasia of the fetal islet cells. ${ }^{11}$ The newborn infants of diabetic mothers (GDM \& PGDM) undergo a sudden interruption of glucose delivery in the postpartum period which, when accompanied by high neonatal insulin levels, results in neonatal hypoglycemia. The effect of neonatal hypoglycemia on the developing brain, with the potential for long term damage is of great concern. ${ }^{12}$ Hypoglycemia is known to be associated with brain dysfunction \& retarded motor development. ${ }^{13}$ Thus hypoglycemia is directly related to neonatal mortality \& sometimes with long term morbidity which can be easily prevented if detected in the early periods of life.

Neonatal hypocalcemia has been reported upto $50 \%$ of IDM (GDM \& PGDM). ${ }^{14}$ The aetiology of the hypocalcaemia is multifactorial \& include prematurity, birth asphyxia, reduce placental transfer of calcium due to substantial maternal urinary excretion of calcium, decrease neonatal secretion of PTH and limited intake \& impaired absorption of ingested calcium. ${ }^{15}$ Transient neonatal hypocalcemia has been mainly reported in neonates of pregestational insulin dependent- diabetic mothers and may be partly related to maternal hypomagnesemia and subsequent fetal hypomagnesemia. The severity of hypocalcemia also appeared to be related to the severity of maternal diabetes, as calcium concentration in the neonates was negatively related to maternal $\mathrm{HbA} 1 \mathrm{c}$ levels. ${ }^{16}$
Infants of both GDM and PGDM are at risk of polycythemia and possibly hyperviscosity syndrome in the neonatal period. Studies suggest that fetal hyperinsulinemia and fuctuation in fetal glucose concentrations may affect fetal oxygen availability, thereby possibly increasing erythropoiesis. Hyperbilirubinemia is extremely common in IDMs (GDM \& PGDM), in part because of their tendency to have high red cell mass. ${ }^{17}$

Though metabolic and hematologic abnormalities are common in both infants of GDM and PGDM but findings are not unanimous. Some author found higher incidence of these abnormalities in infants with GDM group and other founds higher incidence of these abnormalities in infants with PGDM group. The incidence of hypoglycaemia in infants of PGDM ranges from $14.5 \%$ to $42 \%$ and in case of infants of gestational diabetic mother is $12.9 \%$ to $59.4 \%$. ${ }^{18-20}$ If mothers with PGDM had good glycemic control as they were diagnosed before pregnancy and were advised regarding the complications during pregnancy which probably prevent fetal hyperinsulinism and significant hypoglycemia.

The incidence of hypocalcemia in infants of PGDM ranges from $24 \%$ to $25 \%$ and in case of gestational diabetic mothers is $6.7 \%$ to $17.4 \%$. $^{19,20}$ The incidence of polycythaemia in infants of GDMs ranges from $25.8 \%$ to $49.2 \%$ as compared to infants of PGDMs is 9.5 to $11.5 \%{ }^{18,20}$ The incidence of hyperbilirubinemia in infants of PGDMs ranges from $29 \%$ to $33.3 \%$ and in case of gestational diabetic mothers is $31.8 \%$ to $49.3 \% .{ }^{19,20}$

Worldwide several studies done over the infants of diabetic mothers to see the metabolic and haematological profile, but there is scarcity of data regarding these profiles in infant of GDM and PGDM diabetic mothers in Bangladesh. The present study focuses on the consequence of neonates born to diabetic mothers with special reference to their metabolic and hematological profile and to compare the profile between the two groups (GDM \& PGDM) which will help to reduce neonatal mortality \& morbidity.

\section{Materials and Methods:}

The hospital based, prospective observational study was conducted in Dr. M. R. Khan Shishu Hospital \& ICH from January 2018 to December 2018 over a period of one year.Approval from the Institutional Ethical Committee was taken before starting the 
study. Sixty-eight infants born to diabetic mothers, who were admitted at the special care neonatology unit, were included in the study. Infants of GDM and PGDM were categorized by taking history and review of previous records. Neonates of diabetic mothers with medical complications such as chronic heart disease and chronic renal disease and neonates of diabetic mothers with pregnancy induced hypertension (preeclampsia) or eclampsia were excluded from the study. Informed written consent was obtained from parents/ caregivers prior to enrollment of their babies in the study.Status of glycemic control of mother was determined by taking history.

Of all neonates included in the study, detailed examination was performed by the on-duty medical officer at the time of admission and then daily during hospital stay and finally at time of discharge from hospital. Birth weight of each baby was recorded by digital baby weighing scale and gestational age was calculated from New Ballard's gestational scoring chart, subsequently they were grouped as Appropriate for Gestational Age (AGA), Large for Gestational Age (LGA) and Small for Gestational Age (SGA) by plotting their weight and gestational age on Lubchenco charts. Immediately after admission all neonates underwent investigations like complete blood count, metabolic profile including blood sugar, serum calcium. Wherever indicated, serum bilirubin, $X$-ray chest, $A B G$ analysis \& hematocrit was done on need basis. Blood sugar was done immediately after admission and then 6 hourly for 1 st 24 hours of life followed by 12 hourly upto 72 hours of life. Blood sugar was done by Glucostick method \& any abnormal values if found were sent to laboratory of Dr. M. R. Khan Shishu Hospital for confirmation by glucose oxidase method. If any abnormality was found, it was managed as per unit protocol.

Hypoglycemia was defined as blood glucose level below $2.6 \mathrm{mmol} / \mathrm{l}$, while hypocalcemia was defined as a total serum calcium concentration below $7 \mathrm{mg} /$ dl. ${ }^{21}$ Polycythemia was defined as venous hematocrit level $65 \%$ or more and hyperbilirubinemia was defined according to Consensus based bilirubin threshold chart for management of hyperbilirubinemia. ${ }^{21}$

All data was recorded systematically in preformed data collection form and quantitative data was expressed as mean and standard deviation and as numbers (\%) for categorical data. Statistical analyses of the results were obtained by using window based computer software devised with Statistical Packages for Social Sciences (SPSS-23). Statistical tests for significance of difference were done using Chi square test. A 'p' value $<0.05$ was considered as significant.

\section{Results:}

Of the 68 infants of diabetic mothers who were admitted during the study period, $61.8 \%$ (42/68) were born to mothers with GDM, while the remaining $38.2 \%(26 / 68)$ were born to mothers with PGDM. Majority of the neonates (55.9\%) included in the study were males. Lower segment cesarean section was the mode of delivery in $79.4 \%$ (54/68) of neonates. The glycemic control of mother was done by drugs (Insulin and $\mathrm{OHA}$ ) for $58.8 \%$. Majority of the neonates $(76.5 \%)$ were term. (Table I).

Table I

Baseline characteristics of infants of diabetic mother $(n=68)$

\begin{tabular}{llr}
\hline General characteristics & & Number (\%) \\
\hline Diabetic status of mother & GDM & $42(61.8 \%)$ \\
& PGDM & $26(38.2 \%)$ \\
Gender of neonate & Male & $38(55.9 \%)$ \\
& Female & $30(44.1 \%)$ \\
Mode of delivery & NVD & $14(20.6 \%)$ \\
& LSCS & $54(79.4 \%)$ \\
Glycemic control of & Diet and & $28(41.2 \%)$ \\
mother & exercise & \\
& Drugs & $40(58.8 \%)$ \\
Gestational age & Term & $52(76.5 \%)$ \\
& Preterm & $16(23.5 \%)$ \\
\hline
\end{tabular}

In case of GDM $4.8 \%$ were SGA, $61.9 \%$ were AGA and $33.3 \%$ were LGA. In case of PGDM the proportion of SGA, AGA and LGA were $15.4 \%, 61.5 \%$ and $23.1 \%$ respectively. (Table II)

Table III

Table II

Frequency of neonates based on weight for gestational age ( $n=68)$

\begin{tabular}{lccc}
\hline $\begin{array}{l}\text { Diabetic status of } \\
\text { mother }(n=68)\end{array}$ & $\begin{array}{c}\text { Small for gestational } \\
\text { age (SGA) } \\
\text { Number }(\%)\end{array}$ & $\begin{array}{c}\text { Appropriate for } \\
\text { gestational age (AGA) } \\
\text { Number (\%) }\end{array}$ & $\begin{array}{c}\text { Large for gestational } \\
\text { age (LGA) } \\
\text { Number }(\%)\end{array}$ \\
\hline GDM $(42 / 68)$ & $2(4.8 \%)$ & $26(61.9 \%)$ & $14(33.3 \%)$ \\
PGDM $(26 / 68)$ & $4(15.4 \%)$ & $16(61.5 \%)$ & $6(23.1 \%)$ \\
\hline
\end{tabular}


Distribution of metabolic abnormalities in IDMs $(n=68)$

\begin{tabular}{lccc}
\hline $\begin{array}{l}\text { Metabolic } \\
\text { abnormality }\end{array}$ & $\begin{array}{c}\text { Diabetic status } \\
\text { of mother }(n=68)\end{array}$ & $\begin{array}{c}\text { Number } \\
(\%)\end{array}$ & $\begin{array}{c}p \\
\text { value }\end{array}$ \\
\hline Hypoglycemia & GDM $(n=42)$ & $30(71.4 \%)$ & $0.01^{*}$ \\
& PGDM $(n=26)$ & $14(53.8 \%)$ & \\
Hypocalcemia & GDM $(n=42)$ & $20(47.6 \%)$ & $0.02^{*}$ \\
& PGDM $(n=26)$ & $18(69.2 \%)$ & \\
\hline
\end{tabular}

${ }^{*}$ Chi square test was done to measure the level of significance

Hypoglycemia was the most common metabolic abnormality seen in $71.4 \%$ (30/42) of neonates with GDM and $53.8 \%(14 / 26)$ of neonates with PGDM. Other metabolic abnormality like hypocalcemia was seen in $47.6 \%$ (20/42) of neonates with GDM and $69.2 \%(18 / 26)$ of neonates with PGDM. Incidence of hypoglycemia was significantly higher in neonates born to mothers with GDM ( $p$ value 0.01 ) and incidence of hypocalcemia was significantly higher in neonates born to mothers with PGDM ( $p$ value 0.02 ) (Table III). Polycythemia was seen in $33.3 \%$ (14/42)of neonates with GDM and $23.1 \%(6 / 26)$ of neonates with PGDM and hyperbilirubinemia was seen in $66.7 \%(28 / 42)$ of neonates with GDM and $38.5 \%$ (10/26) of neonates with PGDM. Incidence of polycythemia and hyperbilirubinemia were significantly higher in infants of mothers with GDM ( $p$ value 0.02 and $<0.001$ respectively) (Table IV).

\section{Table IV}

Distribution of hematological abnormalities in IDMs

\begin{tabular}{lccc}
\hline $\begin{array}{l}\text { Hematological } \\
\text { abnormality }\end{array}$ & $\begin{array}{c}\text { Diabetic status } \\
\text { of mother }(n=68)\end{array}$ & $\begin{array}{c}\text { Number } \\
(\%)\end{array}$ & $\begin{array}{c}p \\
\text { value }\end{array}$ \\
\hline Polycythemia & GDM $(n=42)$ & $14(33.3 \%)$ & $0.02^{*}$ \\
& $\operatorname{PGDM~}(n=26)$ & $6(23.1 \%)$ & \\
Hyperbilirubinemia & GDM $(n=42)$ & $28(66.7 \%)<0.001^{*}$ \\
& $\operatorname{PGDM~}(n=26)$ & $10(38.5 \%)$ & \\
\hline
\end{tabular}

${ }^{*}$ Chi square test was done to measure the level of significance

\section{Discussion:}

Diabetes has long been associated with maternal and perinatal morbidity and mortality. ${ }^{22}$ In the present study, metabolic and hematological profile of IDMs was studied. These parameters were also compared between IGDMs and IPGDMs and found significant differences in the consequences of neonates. In the present study, majority of neonates were delivered by cesarean section. Most of the cesarean sections were done on emergency basis due to obstetric indications as a result of which the number of neonates delivered by cesarean section was slightly higher than those reported in previous studies. ${ }^{23,24}$ Most of these neonates were delivered at term and were appropriate for gestational age. Similar observation was seen in the study conducted by Mahmood CB et al. ${ }^{18}$ However, in the study conducted by Ranade et al. the proportion of preterm deliveries was higher and was probably because they included all diabetic mothers with coexisting medical complications like $\mathrm{PIH}$, chronic heart and renal disease. ${ }^{24}$

In the present study, frequency of hypoglycemia was significantly higher in IGDM. This observation was in contrast to the results obtained in studies done earlier where the incidence of hypoglycemia was higher in IPGDMs. ${ }^{18,19}$ In the present study, most of the mothers with PGDM had good glycemic control which probably prevented fetal hyperinsulinism and significant hypoglycemia. Glycemic control was better in mothers with PGDM may be due to the fact that, mothers with PGDM were diagnosed before pregnancy and were advised regarding the complications during pregnancy and possible neonatal outcome which enabled strict glycemic control with regular follow up.

It is also important to note that in the present study most of the hypoglycemic neonates were asymptomatic probably because of early introduction of frequent feeds. In the present study, frequency of hypocalcemia was significantly higher in babies born to mothers with PGDM. This may be due to reduced placental transfer of calcium due to substantial maternal urinary excretion of calcium in PGDMs who had poor diabetic control. Similar observation was seen in the study conducted by Nili Firouzeh et al. ${ }^{19}$

Neonatal jaundice (Hyperbilirubinemia) developed in $66.7 \%$ of neonates with GDM and $38.5 \%$ of neonates with PGDM, all these babies were treated with phototherapy except 1 cases which required double volume exchange transfusion. Similarly, polycythemia occurred in $33.3 \%$ of neonates with GDM and $23.1 \%$ of neonates with PGDM, each of them were asymptomatic. Deorari AK et al.in their analysis of 263 infants born to diabetic mothers found that polycythemia occurred in $11 \%$ of babies. ${ }^{25}$ 
In the present study, incidence of polycythemia and hyperbilirubinemia was significantly higher in IGDMs. Similar observation was seen in the studies conducted by Mohsin et al. ${ }^{20}$ But these findings are contrast to the studies done by Deorari AK et al and Firouzeh $\mathrm{N}$ et al; they did not find any statistically significant difference between the two groups. ${ }^{25,26}$

\section{Conclusion:}

Infants born to diabetic mothers remain at high risk for development of metabolic and haematological complications. Hypoglycemia and hypocalcemia remain the most common biochemical abnormality followed by haematological abnormality like polycythemia and hyperbilirubinemia. Among the pregnancies complicated by diabetes, GDM continues to have a major contribution. The frequency of hypoglycaemia, polycythemia and hyperbilirubinemia were significantly more in newborns with GDM compared to infants of PGDM but the frequency of hypocalcemia was significantly more in newborns with PGDM compared to infants of GDM. Adequate glycemic control before and during pregnancy is crucial to improve the outcome of neonates born to diabetic mothers.

\section{References:}

1. Dutta DC. Diabetes mellitus in pregnancy. In: Dutta DC, editor. The text book of Obstetrics, 6th edition. Calcutta: New central book agency; 2006. p.289-90.

2. Cunningham, Leveno, Bloom, Hauth, Rouse, Spong. Diabetes. In: Williams,editor. Obstetrics, 23rd edition. Mc Graw Hill;2010. p. 1104-26.

3. Ang C, Howe D, Lumsden M. Diabetes. In: James DK, Steer PJ, Weiner CP, Gonik B, editors. High Risk Pregnancy Management options, 3rd edition. Philadelphia: Saunders; 2005. p.986-1004.

4. Ferrara A. Increasing prevalence of gestational diabetes mellitus: a public health perspective. Diabetes Care 2007; 302: 141-6.

5. Seshiah V, Balaji V, Balaji MS, Paneerselvam A, Arthi T, Thamizharasi $\mathrm{M}$ etal. Prevalence of gestational diabetes mellitus in South India (Tamil Nadu)- A community based study. J Assoc Physicians India. 2008; 56: 329-33.

6. International Diabetes Federation. Diabetes Atlas. 7th ed. Brussels: International Diabetes Federation; 2015. p 9-25.

7. Sayeed MA, Mahtab H, Khanam PA, Begum R, Banu A, Azad Khan AK. Diabetes and hypertension in pregnancy in a rural community of Bangladesh: a population-based study. Diabet Med 2005;22:1267-71.

8. Jesmin S, Akter S, Akashi H, Al-Mamun A, Rahman MA, Islam MM, et al. Screening for gestational diabetes mellitus and its prevalence in Bangladesh. Diabetes Res Clin Pract 2014;103:57-62.
9. Jesmin S, Jahan S, Khan MI, Sultana N, Jerin J, Habib $\mathrm{SH}$, et al. The incidence, predisposing factors, complication and outcomes of preeclampsia in diabetes pregnancy. Birdem Med J. 2011;1:10-4.

10. Nold JL, Georgieff MK. Infants of diabetic mothers. Pediatr Clin North Am 2004; 51: 619-37.

11. Creasy RK, Resnik R. Intrauterine Growth Restriction. In: Creasy RK, Resnick R, editors. Maternal-Fetal Medicine, 4th ed. Philadelphia: WB Saunders; 1999. p.569-84.

12. Menni F, de Lonlay P, Sevin C, Touati G, Peigne C, Barbier $V$, et al. Neurologic Outcomes of 90 Neonates and Infants with Persistent Hyperinsulinemic Hypoglycemia. Pediatrics. 2001; 107:476-79.

13. World Health Organization. Hypoglycemia of the Newborn: Review of the Literature. 1997 [cited 2004 Feb 25]:http:// apps.who.int/iris/bitstream/10665/63362/1/ WHO_CHD_97.1.

14. Mimouni F, Tsang R C, Hertzberg V S, Miodovnik. Polycythemia, Hypomagnesimia and Hypocalcemia in infants of Diabetic mothers. American Journal of Diseases of children 1986; 140:798-800.

15. Kovacs CS, Kronenberg HM. Maternal-fetal calcium and bone metabolism during pregnancy, puerperium, and lactation.Endocrine Reviews 1997; 18:832-72.

16. Demarini S, Mimouni F, Tsang RC, Khoury J, Hertzberg V. Impact of metabolic control of diabetes during pregnancy on neonatal hypocalcemia: a randomized study. Obstet Gynecol 1994; 83: 918-22

17. Edward S. Ogata, Problems of the Infant of the Diabetic Mother. Article endocrinology. November 2010;11:627-30

18. Mahmood CB, Kayes MI. Problems and immediate outcome of infant of diabetic mother. J Bangladesh Coll Phys Surg 2008; 26: 67-72.

19. Nili Firouzeh, Mahdaviani. Comparision of morbidities between infants of pregestational and gestational diabetic mothers. MJIRI 2004; 18: 13-19.

20. Girish Gopal. A study of clinical, metabolic and hematological profile in infants of diabetic mothers. Indian Journal of Pharmaceutical and Biological Research, 2014; 2: $34-40$.

21. Janet M. Rennie. Metabolic and Endocrine Disorders. In: Jane M Hawdon, Tim Cheetham, Daniel J Schenk, editors. Rennie \& Roberton's Textbook of Neonatology, $5^{\text {th }}$ edition. Churchill Lvingstone: Elsevier; 2012. p.678-83

22. Eleen E Tyrala. Infant of the diabetic mother. Obstet Gynecol Clin North Am 1996; 23: 221-241.

23. Farooq MU, Ayaz A, Ali Bahoo L, Ahmad I. Maternal and Neonatal Outcomes in Gestational Diabetes Mellitus. Int J Endocrinol Metab 2007; 3: 109-15.

24. Ranade, Merchant AY, Bajaj RH, Joshi RT. Infants of diabetic mothers-an analysis of 50 cases, Indian Pediatr. 1989; 26: 366-70.

25. Deorari AK, Kabra SK, Paul VK Singh M. Perinatal outcome of infants born to diabetic mothers. Indian Pediatr. 1991;28:1271-5.

26. Firouzeh N, Mahdaviani. Comparision of morbidities between infants of pregestational and gestational diabetic mothers. MJIRI. 2004;18:139. 